\title{
Structure of HIV-1 CA Protein: Image Reconstruction from Helical Assembly
}

\author{
Gongpu Zhao, Xin Meng, Peijun Zhang \\ Department of Structural Biology, School of Medicine, University of Pittsburgh, Pittsburgh, PA \\ 15260
}

The type 1 human immunodeficiency virus (HIV-1) CA protein can form a conical capsid that surrounds RNA genome, which is crucial for infectivity. In vitro, CA proteins can assemble into cones, spheres and helical tubes [1]. The HIV-1 CA proteins are composed of two domains. The $\mathrm{N}$-terminal domain (NTD) has seven $\alpha$ helices and an amino-terminal $\beta$-hairpin and the Cterminal domain (CTD) has a single-turn $3_{10}$-helix and four short $\alpha$ helices $[2,3]$. Two assembled HIV-CA structures have been reported to date [4,5]. Both of these were derived from cryoEM reconstructions of assembled full-length HIV-1 CA proteins. In both studies, the CA protein carried mutations to stabilize the $2 \mathrm{D}$ or helical assemblies to facilitate structural analysis. These structures have revealed the hexameric HIV-CA organization in the capsid, yet some of the molecular interfaces are inconsistent between these models, particularly at the NTD-CTD interfaces. The density map obtained from 2D crystals formed by flattened spheres [5] showed that there is extensive interaction between NTD and CTD within CA molecule. It was suggested that the helix 4 from NTD inserts into a groove formed by helices 8, 9 on one side and helices 10, 11 on the other side within CTD. The density map reconstructed from helical assembly showed no such interaction and suggested NTD and CTD are only connected through a flexible linker [4]. The discrepancy is likely owing to different forms of assemblies with different mutations, and limited or anisotropic resolution of these maps. Because the structure of the capsid lattice has important implications for our understanding of capsid assembly and disassembly, a reliable high resolution structural model of assembled HIV-CA is necessary. Here we used cryoEM and three-dimensional reconstruction of helical assembly of $\mathrm{CA}$ protein to obtain high resolution structure. HIV-1 CA protein with single point A92E mutation can form long and straight helical tubes. Micrographs with well-ordered long tubes (FIG. 1a) are selected for image processing. The helical symmetry was determined manually from calculated Fourier transforms (FIG. 1b) and a three-dimensional density map was reconstructed using a real space helical processing package IHRSR [6] (FIG. 1c and 1d). The initial reconstructed density map at 20- $\AA$ resolution from one tube clearly shows the hexametric rings formed by the neighboring NTDs. These hexametric rings are connected to the neighbor units through dimerization of underlying CTDs. The connection between NTD and CTD within CA monomer is also visible. A high resolution map, by averaging more tubes, is expected to allow building a reliable pseudo-atomic model of HIV-CA assemblies and to provide insight into the specific intra- and inter- molecular interfaces involved in the core structure. 
References:

[1] B.K. Ganser-Pornillos et al., J. Virol. 78 (2004) 2545.

[2] C. Berthet-Colominas et al., EMBO J. 18 (1999) 1124.

[3] T. R. Gamble et al., Science 278 (1997) 849.

[4] S. Li et al., Nature 407 (2000) 409.

[5] B. K. Ganser-Pornillos et al., Cell 131 (2007) 70.

[6] E. H. Egelman, Ultramicroscopy 85 (2000) 225.
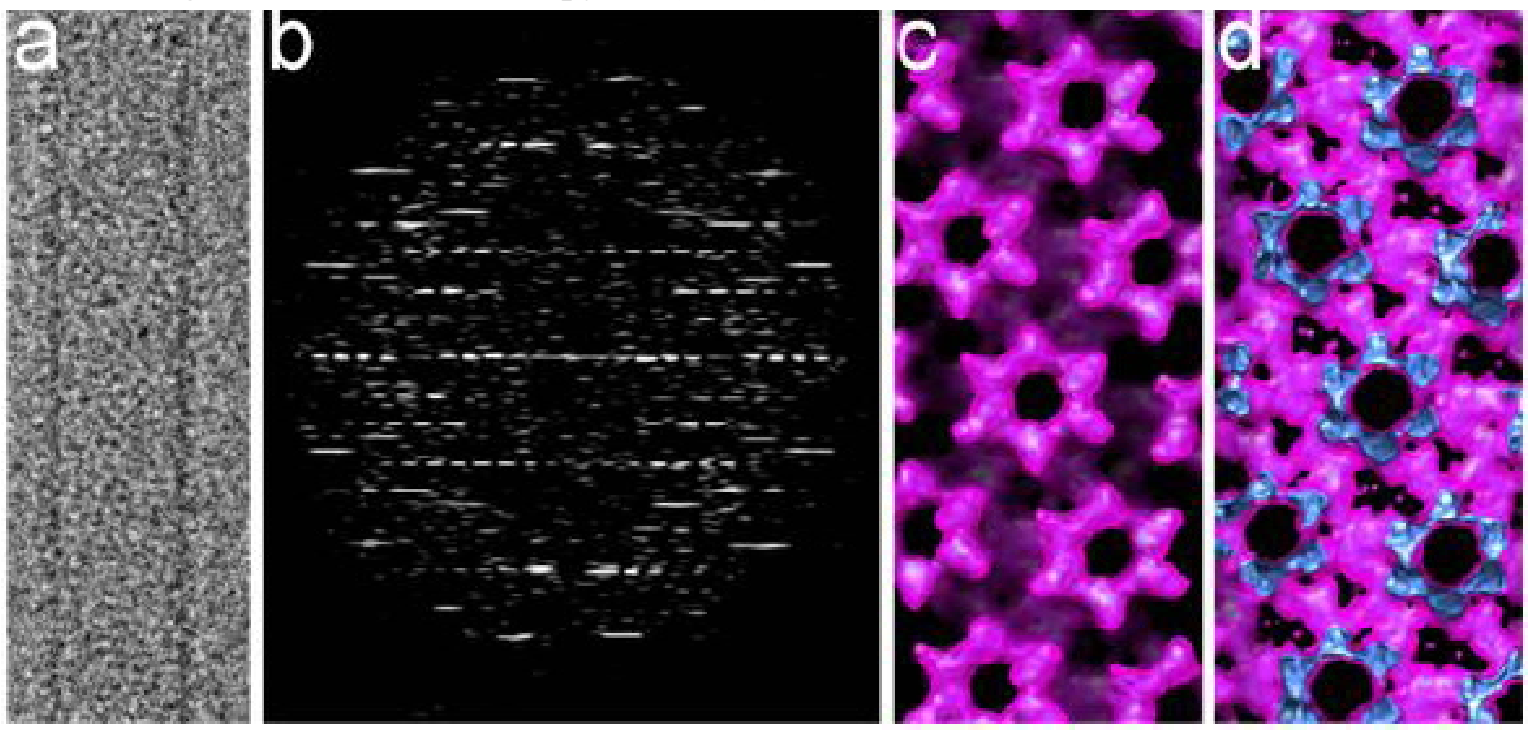

FIG 1. CryoEM and helical reconstruction of CA A92E helical assembly. (a-b) A representative projection image and its Fourier transform. (c\&d). Surface rendering of the density map at the NTD (c) and CTD (d) regions viewed vertical to tube axis. Maps are contoured in magenta for lower contour level and blue for higher contour level. 